\title{
Th1 and Th2 cytokine profile of CD4 and CD8 positive peripheral blood lymphocytes in nickel contact dermatitis
}

\author{
ADEL ALMOGREN $N^{\prime}$ MUSTAFA HUSSEIN ADAMㄴ, ZAHID SHAKOOR ${ }^{l}$, \\ MOHAMMAD OSMAN GADELRAB ${ }^{l}$, HASSAN ABDULAZIZ MUSA ${ }^{3}$
}

${ }^{1}$ Department of Pathology, College of Medicine and University Hospitals, King Saud University, Riyadh, Kingdom of Saudi Arabia ${ }^{2}$ Department of Pathology, King Khalid University Hospital, King Saud University, Riyadh, Kingdom of Saudi Arabia ${ }^{3}$ Faculty of Medicine, National Rabat University, Al Rebat, Khartoum, Sudan

\begin{abstract}
A vast majority of studies investigating cytokine profiles in contact dermatitis $(C D)$ due to nickel have focused on cytokine release in response to nickel challenge. This study was performed to determine the Th1 and Th2 cytokine profile of un-stimulated circulating Tlymphocytes in CD patients with positive nickel skin patch test. A total of 35 patients with positive nickel patch test presenting clinically with CD in allergy clinic at King Khalid University Hospital, Riyadh, were included in the study. Using lysed whole blood method peripheral blood T lymphocytes were analyzed for surface markers and intracellular cytokines by flowcytometry. The proportion of $C D 4+I L-10+$ lymphocytes in the patients $(3.3 \pm 1.1 \%)$ was significantly less than $(p \leq 0.000001)$ the normal controls $(8.4 \pm 2.3 \%)$. The percentage of IL-4+CD4+ lymphocytes in the patients $(1.9 \pm 1.9 \%)$ was also less $(p \leq 0.007)$ than that of the normal controls $(3.1 \pm 1.4 \%)$. For CD8+ lymphocytes nickel sensitive CD patients had a remarkably low ( $p \leq 0.00001)$ percentage of IL-10+ cells then the normal individuals $(1.1 \pm 1.6 \%$ versus $3.3 \pm 1.5 \%)$. The proportion of $C D 8+I L-4+$ lymphocytes was also lower $(p \leq 0.0005)$ in patients $(0.42 \pm 0.41)$ compared to the normal healthy individuals $(0.94 \pm 1.9 \%)$. There was no difference in the percentages of $I F N-\gamma+$ and IL-5+ lymphocytes between the patients and controls. Percentages of IL-10+ and IL-4+ $C D 4+$ lymphocytes $(r=-0.758$ and -0.653 respectively) and $C D 8+I L-10+(r=-0.733)$ cells negatively correlated with patch test scores. These findings indicate that in the healthy state Th2 cytokine profile prevails however lymphocyte cytokine profile is skewed towards Th1 type in patients with nickel contact dermatitis.
\end{abstract}

Key words: nickel, IL-10, IL-4, IFN- $\gamma$, contact dermatitis, Th1, Th2, CD4, CD8.

(Centr Eur J Immunol 2013; 38 (1): 100-106)

\section{Introduction}

Nickel sensitization is common and its prevalence is increasing [1] most likely due to nickel being a highly reactive element and its very wide presence in daily life [2]. Hypersensitivity reactions due to nickel have been reported more frequently among females $(11.1 \%)$ compared to males $(2.2 \%)$ in a population based study [3]. This was also evident in a study examining patch test reactivity in patients suspected to have nickel dermatitis where $0.5 \%$ to $8.5 \%$ of males and $43 \%$ females reacted to epicutaneous nickel patch test [4]. Younger age appears to favor nickel sensitization and development of contact dermatitis (CD) most likely due to an early and prolonged exposure to nickel containing materials such as ear rings [5, 6]. Implementation of legal restrictions on wearing earrings with high nickel content decreased nickel induced dermatitis by $64 \%$ among girls [7]. Nickel sensitization because of the prolonged and direct exposure has been well documented both in the occupational and non-occupational settings [8,9].

Correspondence: Adel Almogren, Department of Pathology, College of Medicine and University Hospitals, King Saud University, Riyadh, Kingdom of Saudi Arabia, phone: 00-966-1-4671843, fax 00-966-1-4671842, e-mail: almogren@ksu.edu.sa 
Nickel induced CD is believed to be a delayed type of hypersensitivity reaction mediated by $\mathrm{T}$ lymphocytes as a result of exposure to the metal [10]. Nickel as a hapten can bind to extracellular proteins and this complex is presented to helper T lymphocytes in association with MHC class II molecules [11]. Activation of T lymphocytes following exposure to nickel results in a proliferation and release of cytokines promoting inflammation $[12,13]$. Traditionally $\mathrm{T}$ helper 1 (Th1) lymphocytes the main source of interferon $\gamma$ $(\mathrm{IFN}-\gamma)$ have been implicated in nickel sensitization [14, 15]. It was mainly due to this reason that a number of studies in the past focused predominantly on role of IFN- $\gamma$ producing cells in nickel induced CD. In contradiction to these observations sufficient evidence has emerged in the recent past supporting the involvement of both Th1 and Th2 lymphocytes in the induction of CD due to nickel $[16,17]$. In addition the intensity of patch test reactivity in patients with $\mathrm{CD}$ due to nickel has also been shown to be correlated with nickel induced secretion of IL- 5 by peripheral blood mononuclear cells indicating that Th2 lymphocytes may be involved in the pathogenesis of nickel induced CD [18]. Interleukin-10 a Th2 cytokine is well known for its downregulatory effects can be induced by nickel thus inhibiting nickel specific Th1 responses [19, 20]. Because of the conflicting evidence the cytokine profile, the relationship between different cytokines and the involvement of Th1 and Th2 lymphocytes in nickel induced CD remains unclear. This study was performed to examine Th1 and Th2 profile of peripheral blood CD4+ and CD8+ lymphocytes of symptomatic nickel patch test positive $\mathrm{CD}$ patients and normal healthy individuals.

\section{Material and methods}

\section{Study population}

A total of 279 patients were patch tested in allergy clinic at King Khalid University Hospital, Riyadh during the period between 2009 to 2012. Among the patients who underwent patch testing 55 patients who reacted only to nickel sulphate were asked to participate in the study and only 35 consenting individuals were included in the study. This group of patients included 29 females and 6 males with the mean age of $35 \pm 7$ years. The duration of the illness ranged between six months to three and a half years. Among the patients 16 patients had lesions on hands, 09 on the dorsum of feet, 06 on ears and 04 on the abdominal skin. All the patients were suffering from clinically active disease at the time of collection of blood samples. A group of twenty otherwise healthy individuals (16 females and 4 males; mean age $29 \pm 8$ years) with no past history or current symptoms suggestive of allergic disorders and negative patch test reactivity was included in the study as a control group. None of the patients were being treated by local or systemic corticosteroids and there was no history of systemic steroid therapy for at least three months prior to being tested.

\section{Patch test}

Patch test was performed using TRUE Test (Mekos Laboratories AS, Denmark) comprising of a panel of 24 haptens/haptens mix. The test panel was applied on the upper part of the back of the patient on the healthy skin free of acne, scars, dermatitis or any other skin condition that might interfere with the interpretation of the results. The patients were instructed to wear the patch for 48 hours without removing it and to avoid contact with water. Interpretations of the results were performed initially after 48 hours followed by a second evaluation 72-96 hours after the application. This protocol allowed sufficient time for the allergic reactions to have fully developed and for the disappearance of mild irritant reactions. Patients were instructed to report back to the clinic in case of delayed reactions. The interpretation of the results was performed in accordance with the recommendations of the International Contact Dermatitis Research Group (ICDRG) and the North American Contact Dermatitis Group. Severity of patch test reaction was graded from no reaction (-) to a maximum of $3+[19]$.

\section{Phenotypic analysis of peripheral blood lymphocytes}

Expression of cell surface and intracellular markers was performed by flowcyometry using lysed whole blood method.

\section{Cell labeling for surface markers}

Cell labeling was performed using whole blood lysis technique ((BD Simultest ${ }^{\mathrm{TM}}$ IMK Plus). One hundred microliters of heparinized whole blood was dispensed into $5 \mathrm{ml}$ polystyrene round bottom tubes (BD Falcon, Biosciences, Erembodegem, Belgium). Twenty microliters of fluorochromes-conjugated mouse anti-human monoclonal antibodies, CD4 FITC (Fluorescein isothiocynate), CD8 FITC, CD8 PE (Phycoerythrin) and IgG1 FITC/IgG2a PE isotype control antibodies (Becton Dickinson, San Joes, CA, USA) were added to the blood sample and incubated for 15 minutes in the dark at room temperature. One tube containing $100 \mu 1$ of blood sample and $20 \mu 1$ of calcium and magnesium free phosphate buffer saline (PAA Laboratories $\mathrm{GmbH}$ PAA-Strasse 1 Austria) served as a negative control. After the incubation $2 \mathrm{ml}$ of FACS lysing solution (B.D Biosciences, San Jose, CA, USA) was dispensed in the tube and incubated for 10 minutes in the dark at room temperature. Following this, the cells were washed in $2 \mathrm{ml}$ of wash buffer at room temperature the supernatant was decanted and the cells were re-suspended in $500 \mu 1$ of $1 \%$ paraformaldehyde (B.D Biosciences pharmingen, San Diego, CA, USA) in phosphate buffered saline for flowcytometric analysis.

\section{Cell labeling for intracellular markers}

Labeling for the intracellular markers for detection of cytokines was performed after labeling the surface markers and prior to fixation. Brefeldin A $10 \mu \mathrm{g} / \mathrm{ml}$ (Sigma Chemi- 


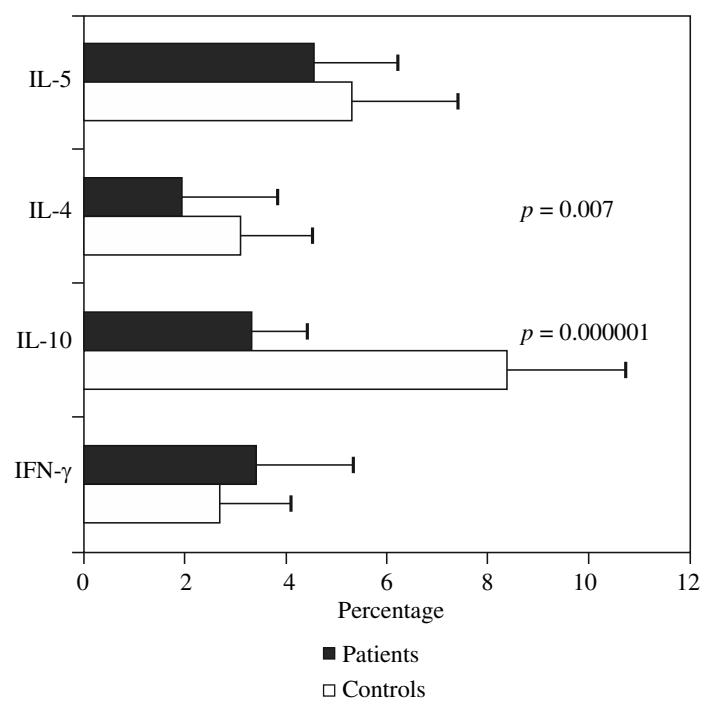

Fig. 1. Cytokine profile of peripheral blood CD4+ lymphocytes in nickel patch test positive patients with contact dermatitis $(n=35)$ and normal controls $(n=20)$

cal) a transport inhibitor that prevents cytokine release from cells was added to the tube. Cells were permeablized by adding $0.5 \mathrm{ml}$ of FACS permeabilizing solution 2 (B.D Biosciences, San Jose, CA, USA) to the sample which was followed by a 10 minute incubation at room temperature. After the incubation the contents of the tube were washed at room temperature with $2 \mathrm{ml}$ of wash buffer containing $10 \mu \mathrm{g} / \mathrm{ml}$ Brefeldin A (Sigma Chemical). The supernatant was decanted and $20 \mu 1$ of each relevant antibody IFN- $\gamma$ PE, IL-4 PerCP (Peridinin chlorophyll protein), IL-5 PE and IL-10 APC (Allophycocyanin) (Becton Dickinson, San Joes, CA, USA) was added and the contents were incubated for $30 \mathrm{~min}$ utes in the dark. Cells were washed the supernatant was decanted and the pallet was finally re-suspended in $500 \mu \mathrm{l}$ of $1 \%$ paraformaldehyde in PBS for flowcytometric analysis.

\section{Flowcytometric analysis}

Labeled samples were analyzed on FACSCalibur (Becton Dickinson, San Jose, CA, USA). In the acquisition mode a total of 10000 events were acquired, and the data were analyzed using Cell Quest software (Becton Dickinson). The negative thresholds were set using isotype-controllabeled cells from both patients and normal controls. Lymphocyte profiling for Th1 and Th2 cells was based on the percentage of lymphocytes labeled for IFN- $\gamma$ and IL-10, IL-4 and IL-5 respectively.

\section{Results}

The majority $(82.9 \%)$ of the patients were females. With regards to patch test scores among the patients $23 \mathrm{had}$ $3+, 3$ had 2+ and 9 had 1+ reactivity for nickel. Based on

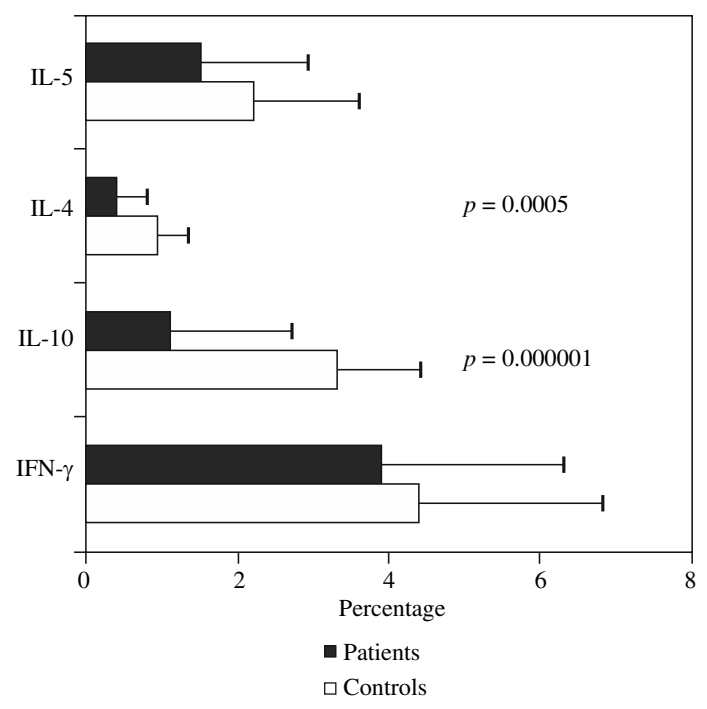

Fig. 2. Cytokine profile of peripheral blood CD8+ lymphocytes in nickel patch test positive patients with contact dermatitis $(n=35)$ and normal controls $(n=20)$

the cytokine labeling Fig. 1 describes Th1 and Th2 profiles of peripheral blood CD4+ lymphocytes in nickel patch test positive CD patients and normal controls. The proportion of CD4+ lymphocytes stained with anti-IL-10 antibody in the patients $(3.3 \pm 1.1 \%)$ was significantly less than $(p \leq 0.000001)$ the normal controls $(8.4 \pm 2.3 \%)$. Similarly the percentage of IL-4 labeled CD4+ lymphocytes in the patients $(1.9 \pm 1.9 \%)$ was also less $(p \leq 0.007)$ than that of the normal controls $(3.1 \pm 1.4 \%)$. No differences were observed between the percentages of CD4+ lymphocytes labeled with IFN- $\gamma(3.4 \pm 1.9 \%$ vs. $2.7 \pm 1.4 \%)$ and IL-5 $(4.5 \pm 1.7 \%$ vs. $5.3 \pm 2.1 \%)$ between patients and normal controls respectively.

Figure 2 compares Th1 and Th2 profile of CD8+ lymphocytes in 35 nickel patch test positive patients with CD and 20 normal healthy individuals. Patients with CD were found to carry a remarkably low $(p \leq 0.00001)$ percentage of CD8+ cells labeled with anti-IL-10 monoclonal antibodies compared to the normal individuals $(1.1 \pm 1.6 \%$ vs. $3.3 \pm 1.5 \%)$. Similarly the proportion of CD8+ cells stained with anti-IL-4 antibodies was lower $(p \leq 0.0005)$ in patients $(0.42 \pm 0.41 \%)$ when compared with the normal healthy individuals $(0.94 \pm 1.9 \%)$. No significant differences were detected in the percentages of cells labeled with IFN- $\gamma(3.9$ $\pm 2.4 \%$ vs. $4.4 \pm 1.9 \%)$ or IL-5 $(1.5 \pm 1.4 \%$ vs. $2.2 \pm 1.5 \%)$ between the patients and the controls respectively.

Correlations were sought between the severity of patch test reactions and the percentages of CD4+ and CD8+ lymphocytes in the peripheral blood from patients with CD (Fig. 3). Linear regression analysis revealed a significant negative correlation between the percentage of IL-10 and IL-4 expressing CD4+ lymphocytes and the nickel patch 


\section{A}
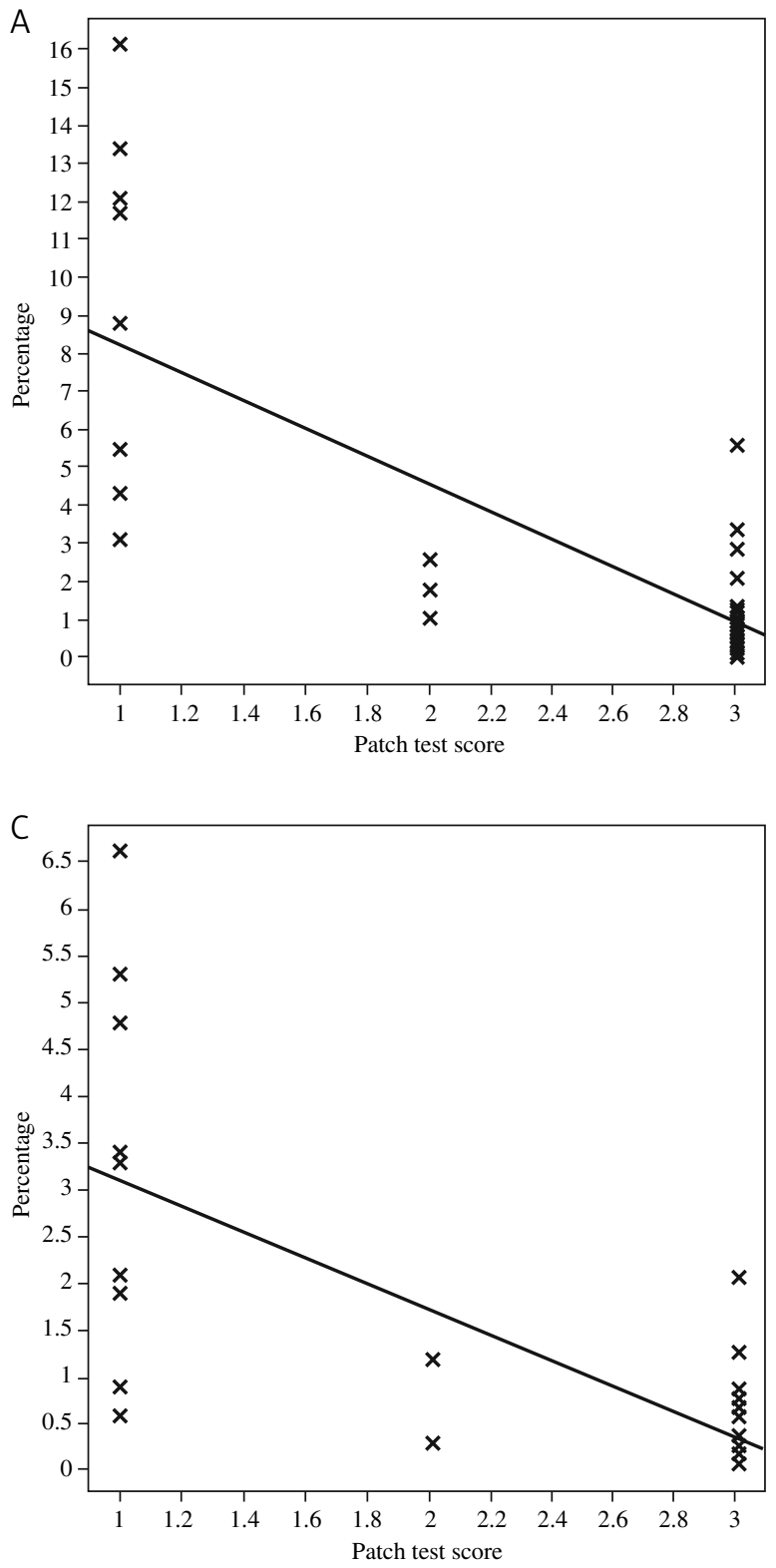

B

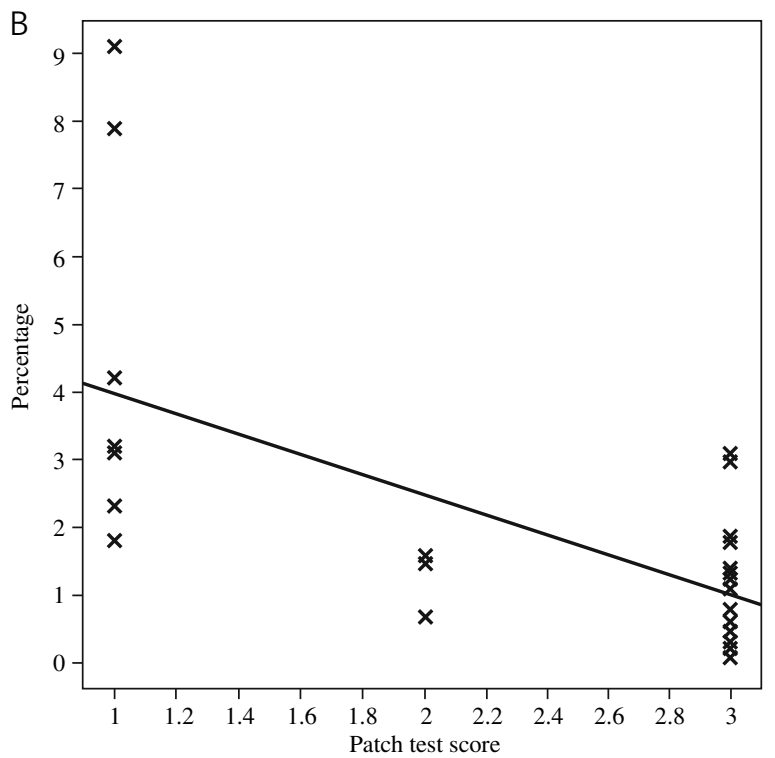

Fig. 3. A) Correlation between percentage of IL-10 producing peripheral blood CD4+ lymphocytes and patch test scores $(r=-0.758)$. B) Correlation between percentage of IL-4 producing peripheral blood CD4+ lymphocytes and patch test scores $(r=-0.653)$; C) Correlation between percentage of IL-10 producing peripheral blood CD8+ lymphocytes and patch test scores $(r=-0.733)$

test scores with the $r$ values of -0.758 and -0.653 Fig. $3 \mathrm{~A}$, $\mathrm{B}$ respectively. For the CD8+ lymphocytes significant negative correlation $(r=-0.733)$ with the patch test reactivity was found only for IL-10 (Fig. 3C).

\section{Discussion}

Whereas a clear deficiency of IL-10 and IL-4 producing CD4+ and CD8+ lymphocytes was evident in nickel patch test positive patients with $\mathrm{CD}$ the percentage of IFN- $\gamma$ expressing lymphocytes though higher in patients failed to achieve statistical significance. These findings indicate that the immune response in nickel allergy is skewed towards Th1 type. Nickel specific CD4+ and CD8+ lymphocytes have been isolated from peripheral blood and the skin lesions and have been shown to be the key cells involved in the epidermal injury in CD [21]. Immunopathogenesis of nickel related $\mathrm{CD}$ is believed to be the result of activation of these nickel specific $\mathrm{T}$ cells leading to their proliferation and production of cytokines [22]. The exact mechanism with regards 
to the involvement of cytokines mediating the inflammatory process in $\mathrm{CD}$ however remains unclear. Traditionally Th1 cytokines particularly IFN- $\gamma$ has been implicated in CD [15] there is however evidence that Th2 cytokines may also be involved [23]. Significantly low numbers of IL-10 and IL-4 producing cells found in nickel patch test reactive patients with CD could be important because of the lack of Th2 inhibitory effect counterbalancing the detrimental effects of Th1 cells [24].

Interleukin 10 formerly described as a Th2 cytokine [25] with regulatory properties has been shown to be produced by cells other than T cells such as monocytes, macrophages and dendritic cells $[26,27]$. This cytokine has recently been shown to be capable of downregulating the production of pro-inflammatory cytokines by Th1 and Th 2 cells in an antigen-specific fashion [28]. In vitro experiments investigating the role of IL-10 in nickel induced IFN- $\gamma$ release by CD4+ cells have clearly demonstrated that neutralization of IL-10 enhances IFN- $\gamma$ production [29]. In addition animal studies involving the induction of nickel tolerance by prolonged oral contact have shown that the key cytokine involved in the nickel tolerance is IL-10. Based on these observations production of IL-10 in response to nickel challenge has been proposed as a test for identification of nickel tolerized individuals [30, 31]. The presence of a significantly higher percentage of IL-10 producing cells in the peripheral blood of patch test negative otherwise normal individuals in the present study is in agreement with the protective role of IL-10 against development of CD. This was also evident in the present study from the fact the intensity of the nickel patch test reaction was negatively correlated with the percentages of IL-10 producing CD4+ and CD8+ lymphocytes in patients with CD.

Patch test negative healthy individuals were also found to harbor a significantly higher percentage of IL-4 a Th2 cytokine producing cells in their peripheral blood when compared with nickel sensitized CD patients indicating a predominant Th2 response in healthy state. Nickel specific $\mathrm{T}$ cell clones derived from the peripheral blood and the skin lesions in CD have been shown differ in their capacity to produce IL-4. Compared to blood, the skin derived nickel specific $\mathrm{T}$ cell clones secrete a significantly higher amount of IL-4 either alone or in combination with IFN- $\gamma$ exhibiting aTh0 cytokine profile indicating compartmentalization of IL-4 secreting cells in nickel sensitized individuals [32]. The detection of decreased percentage of IL-4 producing cells in the peripheral blood of patients with $\mathrm{CD}$ in the present study could possibly be due to increased extravascular migration of IL-4 producing cells in the peripheral tissues. This observation gains further support from the fact that the skin lesions of patients with nickel $\mathrm{CD}$ have been shown to exhibit increased infiltration by Th2 type of cells [33]. Moreover, in vitro Th1 and Th2 skewing of culture conditions using cytokine cocktails have also demonstrated increased nickel specific responses in the presence of Th2 cytokines by peripheral blood cells from patients with nickel contact dermatitis [34]. It is therefore possible that the local pro-inflammatory milieu and cytokine release in the affected tissues skew cellular immune response to Th2 phenotype. In addition serial evaluation of other Th 2 cytokines such as IL-4 and IL-13 release by peripheral blood mononuclear cells in response to nickel challenge have been shown to vary over time in nickel sensitive individuals and exhibit a strong correlation with changes in patch test reactivity [35]. These findings point to the existence of a possible link between the fluctuating systemic $T$ cell reactivity and the changes in disease activity. The fact that all the patients included in the present study had clinically active disease and the observed paucity of IL-4 producing cells in the peripheral blood exhibiting a negative correlation with patch test scores of the patients was probably due to a major shift of IL-4 producing cells to the extravascular compartment.

The involvement of IFN- $\gamma$ and IL-5 in nickel sensitization has been investigated in contact and systemic nickel sensitization (rhinitis and/or diffuse eczema) by challenging peripheral blood mononuclear cells with nickel. Whereas IFN- $\gamma$ was found to be the key cytokine mediating nickel contact dermatitis both IFN- $\gamma$ and IL-5 were proposed to be the principle cytokines mediating systemic allergy [36]. The higher percentage of IFN- $\gamma$ producing cells devoid of statistical significance found in the present study favors a dominant role of IFN- $\gamma$ in nickel CD. These findings may be relevant as the present study compared the cytokine profiles of un-stimulated cells in the peripheral blood of nickel sensitive CD patients. Moreover, it would be interesting to investigate whether IFN- $\gamma$ producing cells from patients with CD differ from normal individuals with regards to their capacity to produce the cytokine in response to nickel challenge. No correlations were detected between the percentages of the IFN- $\gamma$ and IL-5 producing cells in the peripheral circulation with the intensity of patch test reactions. Data regarding such correlations are lacking, a study investigating IFN- $\gamma$ production in response to nickel challenge has also failed to demonstrate any correlation between IFN- $\gamma$ and patch test scores, IL-5 however was shown to exhibit a strong correlation with nickel patch test scores [18]. Nickel has been shown to exhibit cross reactivity to palladium with regards to $\mathrm{T}$ cell recognition of these sensitizing agents [37]. Data from a recent study in agreement with the pervious findings have not only revealed induction of a predominant Th2 response by nickel and palladium challenge but a strong correlation between $\mathrm{Th} 2$ response and skin patch test scores was also observed in individuals sensitized against the respective metals [38]. Along with nickel and palladium other metals such as cobalt, gold and chromium have also been shown to elicit a mixed Th1 and Th2 response in sensitized individuals [39]. Collectively these observations along with the findings of the present study indicate that immune alterations found in patients with nick- 
el induced CD are not nickel specific and may also be observed in individuals sensitized against other metals.

\section{Conclusions}

Majority of the studies investigating Th1 and Th2 cytokines in nickel induced CD have been performed using nickel stimulation assays. This study examining the cytokine profiles of un-stimulated peripheral blood CD4+ and CD8+ lymphocytes describes a predominant $\mathrm{Th} 2$ cytokine profile in normal healthy individuals and Th1 skewed response in patients with nickel induced CD. Because of the conflicting data regarding the cytokine profiles in nickel sensitive individuals large scale studies are needed to further elucidate the role of cytokines in nickel induced CD.

\section{References}

1. Schram SE, Warshaw EM, Laumann A (2010): Nickel hypersensitivity: a clinical review and call to action. Int J Dermatol 49: 115-125.

2. Spiewak R, Pietowska J, Curzytek K (2007): Nickel: a unique allergen - from molecular structure to European legislation. Expert Rev Clin Immunol 3: 851-859.

3. Nielsen NH, Menné T (1992): Allergic contact sensitization in an unselected Danish population. The Glostrup Allergy Study, Denmark. Acta Derm Venereol 72: 456-460.

4. Basketter DA, Briatico-Vangosa G, Kaestner W, et al. (1993): Nickel, cobalt and chromium in consumer products: a role in allergic contact dermatitis? Contact Dermatitis 28: 15-25.

5. Freireich-Astman M, David M, Trattner A (2007): Standard patch test results in patients with contact dermatitis in Israel: age and sex differences. Contact Dermatitis 56: 103-107.

6. Brandao MH, Gontijo B, Girundi MA, et al. (2010): Ear piercing as a risk factor for contact allergy to nickel. J Pediatr (Rio J) 86: 149-154.

7. Jensen CS, Lisby S, Baadsgaard O, et al. (2002): Decrease in nickel sensitization in a Danish schoolgirl population with ears pierced after implementation of a nickel-exposure regulation. Br J Dermatol 146: 636-642.

8. Shah M, Lewis FM, Gawkrodger DJ (1998): Nickel as an occupational allergen. A survey of 368 nickel-sensitive subjects. Arch Dermatol 134: 1231-1236.

9. Klimańska M, Żmudzińska M, Jenerowicz D, Czarnecka-Operacz M (2011): The importance of exposure to contact allergens in patients with allergic contact dermatitis. Postep Derm Alergol 28: 203-211.

10. Grabbe S, Schwarz T (1998): Immunoregulatory mechanisms involved in elicitation of allergic contact hypersensitivity. Immunol Today 19: 37-44.

11. Friedmann PS (2006): Contact sensitization and allergic contact dermatitis: immunobiological mechanisms. Toxicol Lett 162: 49-54.

12. Cavani A, Mei D, Guerra E, et al. (1998): Patients with allergic contact dermatitis to nickel and nonallergic individuals display different nickel-specific $\mathrm{T}$ cell responses. Evidence for the presence of effector CD8+ and regulatory CD4+ T cells. J Invest Dermatol 111: 621-628.

13. Christensen JM, Kristiansen J, Nielsen NH, et al. (2000): Nickel-induced cytokine production from mononuclear cells in nickel-sensitive individuals and controls. Cytokine profiles in nickel sensitive individuals with nickel allergy-related hand eczema before and after nickel challenge. Arch Dermatol Res 292: 285-291.

14. Czarnobilska E, Obtułowicz K, Wsołek K, et al. (2007): Mechanisms of nickel allergy. Przegl Lek 64: 502-505 [article in Polish].

15. Kapsenberg ML, Wierenga EA, Stiekema FE, et al. (1992): Tiggelman AM, Bos JD. Th1 lymphokine production profiles of nickel-specific CD4+ T-lymphocyte clones from nickel contact allergic and non-allergic individuals. J Invest Dermatol 98: 59-63.

16. Probst P, Kuntzlin D, Fleischer B (1995): TH2-type infiltrating $\mathrm{T}$ cells in nickel-induced contact dermatitis. Cell Immunol 165: 134-140.

17. Werfel T, Hentschel M, Kapp A, et al. (1997): Dichotomy of blood- and skin-derived IL-4-producing allergen-specific $\mathrm{T}$ cells and restricted $\mathrm{V}$ beta repertoire in nickel-mediated contact dermatitis. J Immunol 158: 2500-2505.

18. Czarnobilska E, Jenner B, Kaszuba-Zwoinska J, et al. (2009): Contact allergy to nickel: patch test score correlates with IL-5, but not with IFN-gamma nickel-specific secretion by peripheral blood lymphocytes. Ann Agric Environ Med 16: 37-41.

19. Cavani A, Nasorri F, Prezzi C, et al. (2000): Human CD4+ T lymphocytes with remarkable regulatory functions on dendritic cells and nickel-specific Th1 immune responses. J Invest Dermatol 114: 295-302.

20. Spiewak R (2008): Patch testing for contact allergy and allergic contact dermatitis. Open Allergy J 1: 42-51.

21. Traidl C, Sebastiani S, Albanesi C, et al. (2000): Disparate cytotoxic activity of nickel-specific CD8+ and CD4+ T cell subsets against keratinocytes. J Immunol 165: 3058-3064.

22. Borg L, Christensen JM, Kristiansen J, et al. (2000): Nickel induced cytokine production from mononuclear cells in nickel-sensitive individuals and controls. Cytokine profiles in nickel sensitive individuals with nickel allergy-related hand eczema before and after nickel challenge. Arch Dermatol Res 292: 285-291.

23. Minang JT, Troye-Blomberg M, Lundeberg L, et al. (2005): Nickel elicits concomitant and correlated in vitro production of Th1-, Th2-type and regulatory cytokines in subjects with contact allergy to nickel. Scand J Immunol 62: 289-296.

24. Saint-Mezard P, Rosieres A, Krasteva M, et al. (2004): Allergic contact dermatitis. Eur J Dermatol 14: 284-295.

25. Mosmann TR, Schumacher JH, Fiorentino DF, et al. (1990): Isolation of monoclonal antibodies specific for IL- 4, IL-5, IL-6, and a new Th2-specific cytokine (IL-10), cytokine synthesis inhibitory factor, by using a solid phase radioimmunoadsorbent assay. J Immunol 145: 2938-2945.

26. Enk AH, Katz SI (1992): Identification and induction of keratinocytederived IL-10. J Immunol 149: 92-95.

27. Iwasaki A, Kelsall BL (1999): Freshly isolated Peyer's patch, but not spleen, dendritic cells produce interleukin 10 and induce the differentiation of T helper type 2 cells. J Exp Med 190: 229-239.

28. Faith A, Singh N, Farooque S, et al. (2012): T cells producing the anti-inflammatory cytokine IL-10 regulate allergen-specific Th2 responses in human airways. Allergy 2012; 67: 10071013.

29. Minang JT, Areström I, Zuber B, et al. (2006): Nickel-induced IL-10 down-regulates Th1- but not Th2-type cytokine responses to the contact allergen nickel. Clin Exp Immunol 143: 494-502. 
30. Roelofs-Haarhuis K, Wu X, Gleichmann E (2004): Oral tolerance to nickel requires CD4+ invariant NKT cells for the infectious spread of tolerance and the induction of specific regulatory T cells. J Immunol 15: 1043-1050.

31. Rustemeyer T, von Blomberg BM, van Hoogstraten IM, et al. (2004): Analysis of effector and regulatory immune reactivity to nickel. Clin Exp Allergy 34: 1458-1466.

32. Werfel T, Hentschel M, Kapp A, et al. (1997): Dichotomy of blood- and skin-derived IL-4-producing allergen-specific T cells and restricted $\mathrm{V}$ beta repertoire in nickel-mediated contact dermatitis. J Immunol 158: 2500-2505.

33. Probst P, Küntzlin D, Fleischer B (1995) TH2-type infiltrating $\mathrm{T}$ cells in nickel-induced contact dermatitis. Cell Immunol 165: 134-140.

34. Spiewak R, Moed H, von Blomberg BM, et al. (2007): Allergic contact dermatitis to nickel: modified in vitro test protocols for better detection of allergen-specific response. Contact Dermatitis http://www.ncbi.nlm.nih.gov/pubmed/17244072 56: 63-69.

35. Masjedi K, Bruze M, Hindsén M, et al. (2009): Is the variability of nickel patch test reactivity over time associated with fluctuations in the systemic T-cell reactivity to nickel? $\mathrm{Br}$ J Dermatol 161: 102-9.

36. Czarnobilska E, Thor P, Kaszuba-Zwoinska J, et al. (2006): Response of peripheral blood mononuclear leukocyte to nickel stimulation in patients with systemic and contact allergy to nickel. Przegl Lek 63: 1276-1280 [article in Polish].

37. Pistoor FH, Kapsenberg ML, Bos JD, et al. (1995): Cross-reactivity of human nickel-reactive T lymphocyte clones with copper and palladium. J Invest Dermatol 105: 92-95.

38. Muris J, Feilzer AJ, Kleverlaan CJ, et al. (2012): Palladiuminduced Th2 cytokine responses reflect skin test reactivity. Allergy 67: 1605-1608.

39. Minang JT, Areström I, Troye-Blomberg M, et al. (2006): Nickel, cobalt, chromium, palladium and gold induce a mixed Th1- and Th2-type cytokine response in vitro in subjects with contact allergy to the respective metals. Clin Exp Immunol 146: 417-426. 